Project 1021925

\title{
A Phytoremediation Strategy for Arsenic
}

\author{
Meagher, Richard B. \\ University of Georgia
}

\begin{abstract}
RESULTS TO DATE: A Phytoremediation Strategy for Arsenic Progress Report May, 2005 Richard B. Meagher Principal Investigator Arsenic pollution affects the health of several hundred millions of people world wide, and an estimated 10 million Americans have unsafe levels of arsenic in their drinking water. However, few environmentally sound remedies for cleaning up arsenic contaminated soil and water have been proposed. Phytoremediation, the use of plants to extract and sequester environmental pollutants, is one new technology that offers an ecologically sound solution to a devastating problem. We propose that it is less disruptive to the environment to harvest and dispose of several thousand pounds per acre of contaminated aboveground plant material, than to excavate and dispose of 1 to 5 million pounds of contaminated soil per acre (assumes contamination runs $3 \mathrm{ft}$ deep). Our objective is to develop a genetics-based phytoremediation strategy for arsenic removal that can be used in any plant species. This strategy requires the enhanced expression of several transgenes from diverse sources. Our working hypothesis is that organ-specific expression of several genes controlling the transport, electrochemical state, and binding of arsenic will result in the efficient extraction and hyperaccumulation of arsenic into aboveground plant tissues. This hypothesis is supported by theoretical arguments and strong preliminary data. We proposed six Specific Aims focused on testing and developing this arsenic phytoremediation strategy. During the first 18 months of the grant we made significant progress on five Specific Aims and began work on the sixth as summarized below. Specific Aim 1: Enhance plant arsenic resistance and greatly expand sinks for arsenite by expressing elevated levels of thiol-rich, arsenic-binding peptides. Hyperaccumulation of arsenic depends upon making plants that are both highly tolerant to arsenic and that have the capacity to store large amounts of arsenic aboveground. Phytochelatins bind diverse thiolreactive elements like As(III) and are synthesized from amino acids in a three-step enzymatic pathway utilizing three enzymes: ECS = gamma-glutamylcysteine synthetase; GS = GSH synthetase; and PS = phytochelatin synthase. We cloned each of the genes that encode these enzymes and used at least two different plant promoters to express them in transgenic Arabidopsis. We have shown that all three confer significant resistance to arsenic and allow rapid growth on a concentration of arsenic (300 micromolar) that kills wild-type seeds and plants. Manuscripts describing arsenic resistance, processing, and accumulation resulting from strong ECS expression (Li et al., 2005) or strong PCS expression (Li et al., 2004) in transgenic Arabidopsis plants were recently published. A third manuscript on the efficient
\end{abstract} movement of thio-peptides from shoots to roots in plants expressing PCS in shoots alone is in preparation (Li et al., in prep.). The high level of arsenic resistance and sinks for As storage conferred by these genes and peptides are essential to our overall arsenic phytoremediation strategy, which focuses on bringing more arsenic into the plant and storing it aboveground. Specific Aim 2: Convert arsenate to arsenite in aboveground organs by expressing a bacterial arsenate reductase (ArsC) gene under a strong lightregulated leaf-specific promoter. This work was published in Nature Biotechnology and New Phytologist (Dhankher et al., 2002; Dhankher et al., 2003). Specific Aim 3: Characterize the endogenous root-specific plant arsenic reductase, AtACR2, and enhance the transport of arsenate from roots to shoots by knocking down AtACR2 activity. The idea of blocking a theoretical plant arsenic reductase in roots was based almost entirely on analytical data showing very high arsenite levels in plant roots exposed to arsenate. Thus, the potential success of this aim was perhaps the most difficult to predict in our overall arsenic phytoremediation strategy. We were not entirely sure what plant arsenate reductases should look like and if they might not be part of one or more redundant gene families. Based on weak homology to a yeast arsenate reductase ScACR2 and to phosphate transporters, we cloned a possible arsenic reductase from Arabidopsis, AtACR2. Its identity as an arsenic reductase was not completely assured by sequence homology, because of its similar levels of sequence relatedness to two distant classes of enzymes. Since grant submission, we have demonstrated that AtACR2 encodes a true arsenate reductase. AtACR2 suppressed the pleotropic phenotypes of different E. coli mutants defective in the bacterial arsenic reductase arsC gene, as tested with and without the activities of the bacterial arsA/B pump. We prepared an antibody to AtACR2 so we could monitor its expression during transgenic expression experiments. We 
demonstrated that overexpressing the AtACR2 gene in Arabidopsis makes the plants hypersensitive to arsenic. More importantly, knocking down AtACR2 levels using RNA interference (RNAi) caused the plants to accumulate 7 to 17 fold more arsenic in shoots than wildtype. These data and a correlation with AtACR2 expression levels in knockdown plants have been well quantified and submitted for publication to P.N.A.S. (Dhankher and Meagher, in prep.). Arsenic reduction in roots was one of the biggest barriers to our developing a highly efficient phytoremediation strategy for arsenic. In the near future, the knockdown of AtACR2 expression will be combined with factors that will increase the aboveground sinks for arsenic and that will confer arsenic resistance. In order to focus the AtACR2 RNAi knockdown in roots and to express other genes in all root tissues, we have developed a root-specific expression system, based on a bacterial repressor-operator complex or ROC concept. This plant ROC uses the interaction of a shootspecific repressor and a modified, actin ACT2 promoter terminator complex (A2pt), A2pot that contains operators sequences that can be repressed in shoots and remain active in all root cell types. The modified Lac repressor with a nuclear localization signal Lacln is expressed in shoots from the rubisco promoter and terminator in S1pt::IacIn. In more than 25\% of the plants transformed with both the S1pt::Iacln and A2pot::GUS constructs there was strong root GUS expression in all cell types and more than a 1000 fold repression of shoot GUS activity, as compared to plants lacking the repressor. It out performed two other previously published root-specific promoters that we tested. This newly developed root ROC expression system is in press at Plant Biotechnology (Kim et al., in press). Specific Aim 4: Enhance arsenate uptake via the overexpression of high-affinity phosphate transporters. There are at least four ancient classes of high-affinity phosphate transporters in higher plants and at least nine genes encoding them in Arabidopsis. We propose that one class is likely to be better at inadvertent arsenic transport than others, but this may not be one of the classes already expressed in roots, because there may have been an evolutionary selection for root-specific transporters to discriminating against arsenic uptake. In the first year of the grant we recruited a new postdoctoral fellow, Dr. Aaron Smith, and an undergraduate UGA Presidential Scholar, Rebekah Rogers, to work on the project. They cloned and performed in vitro mutagenesis on one phosphate transporter from each of the four clades to make them more suited for subsequent genetic manipulation. All four PHT genes were cloned for plant expression from the constitutive actin ACT2 promoter cassette A2pt, which has strong root and shoot activity. When PHT7 was constitutively expressed in transgenic Arabidopsis, it significantly increased arsenic accumulation in aboveground tissues, while the other transporters did not have as significant an effect. Arabidopsis PHT7 overexpression nearly doubled the arsenic content of Arabidopsis plants. PHT7 will be used in combination with other genes to make healthy plants for arsenic remediation. Next we will perform extensive in vitro mutagenesis to decrease the specificity for phosphate transport and increase the specificity for arsenate transport. Specific Aim 5: Enhance intercellular transport of peptide thiolarsenite complexes into vacuoles in leaf cells by elevating the expression of a glutathione conjugate pump (GCP). We have just begun to work on this specific aim with the cloning of the YCF1 gene from yeast. The YCF1 gene has already been shown to enhance both lead and cadmium transport into yeast vesicles and to generate 1.5 to 1.8 fold increases in $\mathrm{Pb}$ and $\mathrm{Cd}$ uptake by transgenic YCF1 expressing Arabidopsis. We used in vitro mutagenesis to alter YCF1 and make it more suitable for cloning in the root, shoot, and constitutive plant expression systems we use. Once we complete some initial testing in plants we will begin to screen mutant ycf1 libraries for enhanced arsenate uptake and depressed phosphate uptake in yeast. Specific Aim 6: Combine these various transgenic elements into test plants and demonstrate dramatic increases in arsenic resistance and hyperaccumulation aboveground. Our strategy for arsenic phytoremediation is focused on five classes of genes controlling the electrochemical state, transport, and binding of arsenic in plants. Between five to eight transgenes will be needed to optimize arsenic extraction from soil, transport the metalloid to the top of the plant, accumulate thiol-peptidemetalloid conjugates, and transport arsenic into vacuoles for storage. We will test various logical combinations of genes that optimize resistance and hyperaccumulation. We have already shown that combining leaf expressed ArsC with constitutive ECS produced a dramatically high level of arsenic resistance and a moderate three-fold increase in accumulation (Dhankher et al., 2002). In the last year we combined transgenic ECS, GS, and PCS overexpression into one plant line and these plants appear to be much more arsenic resistant than plants with any one or two genes alone. This work will be quantified over the next few months. The speed and simplicity of Arabidopsis genetics makes this possible, but putting 4 to 7 transgenes in a single plant presents many technical difficulties. We have use a method called Critical Path Analysis (CPA) to map out this multistep process. The CPA for the construction of an Arabidopsis As hyperaccumulator with seven stacked transgenes combining the first 
four genes by classical genetics, making plants homozygous for all for single copy transgenes, and then co-transforming in the last three trangenes. Effective immuno assays for three enzymes allows us to efficiently monitor our progress. We are using ligation-mediated PCR to identify the sites (boundary sequences) flanking each transgene insertion so that the homozygous (HOM) plants can be screened out from segregating heterozygotes (HET) by PCR. We plan by the end of the three-year grant period to have engineered plants that accumulate 20 to 50 fold more arsenic in leaf tissue (2000 ppm) than WT when exposed to subtoxic levels of arsenate and we believe this will require a minimum of five to six different transgenes.

DELIVERABLES: References Dhankher OP, Li Y, Rosen BP, Shi J, Salt D, Senecoff JF, Sashti NA, Meagher RB (2002). Engineering tolerance and hyperaccumulation of arsenic in plants by combining arsenate reductase and gamma-glutamylcysteine synthetase expression. Nat. Biotechnol. 20(11): 11405. Dhankher OP, Meagher RB (in prep.) Increased arsenic uptake by plants knocked down for an endogenous arsenate reductase. Dhankher OP, Rosen BP, Fuhrman M, Meagher RB (2003) Increased cadmium tolerance and accumulation by plants expressing bacterial arsenate reductase. New Phytologist 159: 431-441 Kim T, Balish RS, Heaton ACP, McKinney EC, Meagher RB (in revision) Engineering a Root-Specific, Repressor-Operator Gene Complex. Plant Biotech Li Y, Dhankher O, Carreira L, Cobbett $\mathrm{C}$, Meagher R (in prep.) Long-distance transport of thiol-peptides synthesized from the shoot-specific expression of gamma-glutamylcysteine synthetase enhances arsenic tolerance. Li Y, Dhankher O, Carreira L, Lee D, Chen A, Schroeder J, Balish R, Meagher R (2004). Overexpression of phytochelatin synthase in Arabidopsis leads to enhanced arsenic tolerance and cadmium sensitivity. Plant \& Cell Physiol. 45: 1787-1797 Li Y, Dhankher O, Meagher R (2005) Engineered overexpression of gglutamylcysteine synthetase in plants confers high level arsenic and mercury tolerance. Env Tox \& Chem in press 